\title{
REVIEWS
}

\section{Systematic Review and Meta-analysis of the Effectiveness of Implementation Strategies for Non-communicable Disease Guidelines in Primary Health Care}

\author{
Eva Kovacs, Dr. med. PhD ${ }^{1,2}$, Ralf Strobl, Dr. rer. biol. hum., Dipl.-Stat. ' , Amanda Phillips, MSc ",2, \\ Anna-Janina Stephan, MPH ${ }^{1,2}$, Martin Müller, MPH, Prof. Dr. ${ }^{3}$, \\ Jochen Gensichen, Prof. Dr. med. habil., Dipl.-Päd., MPH', and Eva Grill, Prof. Dr., MPH',2,5 \\ IInstitute for Medical Information Processing, Biometrics and Epidemiology, Ludwig-Maximilians-Universität, Munich, Germany; ${ }^{2}$ German Center for \\ Vertigo and Balance Disorders, University Hospital, Ludwig-Maximilians-Universitöt München, Munich, Germany; ${ }^{3}$ Faculty of Applied Health and \\ Social Sciences, Rosenheim University of Applied Sciences, Rosenheim, Germany; ${ }^{4}$ Institute of General Medicine, University Hospital, Ludwig- \\ Maximilians-Universität München, Munich, Germany; ${ }^{5}$ Munich Center of Health Sciences, Ludwig-Maximilians-Universität, Munich, Germany.
}

BACKGROUND: As clinical practice guidelines represent the most important evidence-based decision support tool, several strategies have been applied to improve their implementation into the primary health care system. This study aimed to evaluate the effect of intervention methods on the guideline adherence of primary care providers (PCPs).

METHODS: The studies selected through a systematic search in Medline and Embase were categorised according to intervention schemes and outcome indicator categories. Harvest plots and forest plots were applied to integrate results.

RESULTS: The 36 studies covered six intervention schemes, with single interventions being the most effective and distribution of materials the least. The harvest plot displayed 27 groups having no effect, 14 a moderate and 21 a strong effect on the outcome indicators in the categories of knowledge transfer, diagnostic behaviour, prescription, counselling and patient-level results. The forest plot revealed a moderate overall effect size of 0.22 $[0.15,0.29]$ where single interventions were more effective $(0.27[0.17,0.38])$ than multifaceted interventions $(0.13$ [0.06, 0.19]).

DISCUSSION: Guideline implementation strategies are heterogeneous. Reducing the complexity of strategies and tailoring to the local conditions and PCPs' needs may improve implementation and clinical practice.

KEY WORDS: evidence-based medicine; implementation; general practitioner; intervention; harvest plot.

J Gen Intern Med 33(7):1142-54

DOI: $10.1007 / \mathrm{s} 11606-018-4435-5$

(c) Society of General Internal Medicine 2018

Electronic supplementary material The online version of this article (https://doi.org/10.1007/s11606-018-4435-5) contains supplementary material, which is available to authorized users.

Received March 8, 2017

Revised August 10, 2017

Accepted March 23, 2018

Published online May 4, 2018

\section{BACKGROUND}

Up to $90 \%$ of patient encounters with health professionals occur in the primary health care setting. ${ }^{1,2}$ Performance and quality of primary health care has therefore substantial impact on public health. ${ }^{3,4}$ Although the generalisability of clinical practice guidelines has recently been challenged, ${ }^{5,6}$ they are still important for decision-making in primary care. Guidelines offer a synthesis of the current evidence and recommendations for action. However, their use is still not comprehensively accepted by primary care providers (PCPs). ${ }^{7,8}$ From the first comprehensive framework of facilitators and barriers ${ }^{9}$ to recent progresses in this field, ${ }^{1}$ a wide range of internal and environmental factors were mapped, challenging guideline adherence. Each of these barriers can be addressed by welltargeted intervention methods. ${ }^{10}$ The vast majority of intervention efforts focused on changing the individual behaviour of the practicing professional, e.g. aiming to increase the PCPs' knowledge and/or skills by educational meetings or outreach visits; or to motivate by involving local opinion leaders, or auditing the PCP practice, ${ }^{11-13}$ without convincing breakthrough. External barriers can be managed, e.g. by changing the regulatory environment or the method of financing, thus creating interest in guideline adherence; or by organisational interventions ${ }^{11-13}$ like improving facilities or referral possibilities. However, in spite of all intervention efforts, guideline adherence still cannot be regarded as universal. ${ }^{1,5,7,12-15}$

This systematic review aimed to evaluate the effectiveness of interventions to improve guideline adherence of PCPs in the primary care setting, focusing on recent results.

\section{METHODS}

\section{Eligibility Criteria}

All publications of original research were included if they referred to guidelines of non-communicable diseases for adult ( $\geq 18$ years) patient populations and described an intervention 
performed in the setting of primary health care, targeting the PCP. General quality control measures without direct guideline reference were excluded.

For the sake of comparability, only studies performed in developed countries were considered, according to the categorisation of the United Nations Statistics Division (UNSD). ${ }^{16}$

\section{Search Strategy and Information Sources}

This review followed the PRISMA statement ${ }^{17}$ for systematic reviews. Electronic search was performed in Medline and Embase for studies published between November 2009 and October 2017, focusing on recent results in this field, with the following search strategy: (("Physicians, Family"[Mesh] OR "Family Practice"[Mesh] OR "General Practitioners"[Mesh] OR "General Practice"[Mesh] OR "Primary Health Care"[Mesh]) AND ((guideline*[Title/Abstract]) OR ("Evidence-Based Medicine"[Mesh]) OR ("Practice Guidelines as Topic"[Mesh])) AND (implement* OR disseminat* OR diffusion OR "knowledge translation")) NOT ("Nursing"[Mesh] OR "Developing Countries"[Mesh]).

The inclusion was restricted to those studies which were published in English or German. Full-text papers were retrieved via the library service of the university.

\section{Data Extraction}

Two reviewers (EK and EG) independently screened the retrieved studies for inclusion criteria based on title and abstract. The included studies were assessed likewise based on full text. Agreement on the selection was achieved by consensus. If disagreement could not be resolved by discussion, a third independent researcher (MM) made the decision.

A Microsoft Access-based data extraction form was developed (RS) and pre-tested on five studies (EK and EG). Information regarding the targeted guideline, the characteristics of studies, the applied intervention methods, outcome indicators and results were collected with the help of this form by one reviewer (EK) and randomly tested by the second reviewer (EG). All quality criteria and results were controlled by a second researcher (RS).

\section{Intervention Methods}

We categorised interventions according to the Cochrane Effective Practice and Organisation of Care Group (EPOC) $)^{11}$ taxonomy of interventions (1): professional interventions targeting the health care provider directly, (2) financial interventions addressing either the provider or the patient by various means of incentives, (3) organisational interventions aiming to support the desired behaviour by modifying the setting and (4) regulatory interventions which introduce changes on the level of legislation. The EPOC taxonomy further divides the ${ }^{1}$ professional interventions into (1.1) distribution of educational materials, (1.2) educational meetings,
(1.3) local consensus processes, (1.4) educational outreach visits, (1.5) involvement of local opinion leaders, (1.6) patient-mediated interventions, (1.7) audit and feedback, (1.8) reminders, (1.9) marketing and (1.10) mass media.

Intervention schemes were categorised according to the number of applied intervention methods as a main criterion (i.e. single or multifaceted approach). Further refinement of this categorisation was based on the above types of intervention methods.

\section{Outcome Indicators}

For the classification of the outcome indicators, we followed the method of Grimshaw ${ }^{12}$ and included all measures of the process of care (i.e. the PCPs' activities) and/or the outcome of care (i.e. patient-level results). We further categorised the process of care indicators as prescription, diagnostic behaviour, patient counselling and knowledge level.

\section{Synthesis of the Results}

Harvest Plots. Harvest plots ${ }^{18}$ provide an alternative graphical method for displaying combined data when the complexity of the intervention schemes, the diversity of the applied indicator sets and the inhomogeneity of the outcomes prevent direct comparison using traditional meta-analytic methods such as the forest plot. Harvest plots show both the effect size and other parameters of interest such as study quality.

All outcome indicator categories (both process of care and outcome of care) were included in this analysis. Though some studies indicated main outcome(s) and secondary outcome(s), each of them was handled equally. Outcome indicators within a study were grouped into the outcome categories of prescription, diagnostic behaviour, counselling, knowledge and patient-level outcome of care; thus, a study could report up to five outcome groups. Outcome groups were displayed by bar charts, with each bar referring to one outcome group of a study, so a study could be represented by up to five bars corresponding to the number of the covered outcome categories.

Level of effect was estimated by the proportion of improved outcome indicators and classified as "no effect" (the study has not reported any significant improvement among the outcome indicators), "moderate effect" ( $\leq 50 \%$ of the reported outcome indicators within a category improved significantly) and "strong effect" ( $>50 \%$ of the reported outcome indicators of a category improved significantly). This categorisation was chosen because it reflects the distribution of the proportions and also allows to visualise this outcome in a harvest plot. The categorisation does not consider level of significance or absolute magnitude of effect.

Study quality was assessed by four of the five criteria of the Cochrane risk of bias tool ${ }^{19}$ (random allocation to the interventions, blinding of the outcome assessors, completeness of the outcome data, avoiding selective reporting), except for blinding of participants which is rarely possible in health 
services research and thus commonly left out. ${ }^{20}$ The fulfilment of each of these four criteria was rated as no bias (3 points); most likely no bias ( 2 points); and no info or a reported problem (1 point). Overall study quality was then summarised as the average of the four ratings. Sample size was considered relevant for the reliability of results; however, the actual power of each study was not reported in most of the studies. Thus, instead of power, we chose to display sample size instead, i.e. an artificial threshold of involving at least 100 PCPs was chosen to display more reliable results.

Results were visualised in a matrix where each row corresponds to an intervention scheme and each column to an effect size category. Within each cell of this matrix, bar charts display the two quality parameters of the included studies where the height of each bar indicates the quality of the study and the colour the sample size.

Forest Plot. We included controlled trials and controlled before-after studies with either a dichotomous or continuous outcome. We displayed the main outcome of each study, if explicitly stated in the article. If the main outcome was not clearly defined, we decided to display all outcomes. To make outcomes comparable across studies, effect measures were transformed to a common scale centred to zero, i.e. absence of an intervention effect would be represented by a zero, a beneficial effect of the intervention by a value greater than zero, and a harmful effect by a value less than zero. Due to the heterogeneity of the included studies and their different outcomes, we used a random effects model rather than a fixed effect model to estimate a summary measure of the pooled outcomes. Regarding transformation of effect measures, see details in electronic supplementary material (ESM 1).

For calculating the forest plot and the summary measures, we used the function "rma" from the "metafor"-package ${ }^{21}$ running $\mathrm{R} 3.0 .3{ }^{22}$

To test for asymmetry in the funnel plot, we applied the Egger test. ${ }^{23}$

Data Availability. The dataset analysed during the current study is available from the corresponding author on reasonable request.

\section{RESULTS}

\section{Selected Studies}

We identified 1103 records from the database search, from which 211 records qualified for full-text assessment, 38 records were decided by discussion (EK, EG) and 27 records involved the third researcher $(\mathrm{MM})$ in the decision process. Applying the inclusion criteria, 36 studies were eligible for harvest plot analysis. Among these, 21 were included in the forest plot. A flow chart of study selection according to PRISMA requirements and detailed reasoning for exclusion is displayed in Figure 1.

The studies (Table 1) represented a wide geographical coverage from three continents. Controlled studies $(n=17)$ where randomisation was typically on the cluster level due to the organisational requirements of these types of intervention, and controlled before-after studies $(n=11)$ were included in the analysis. The uncontrolled before-after studies $(n=8)$ were included only in the harvest plot. General quality of the studies was moderate with a median quality score of 2 out of 4 (Table 1). Disagreement regarding quality criteria was solved by discussion in six cases (EK, RS). The funnel plot has not revealed major concern regarding publication bias. Egger's test for asymmetry resulted in a $p$ value of 0.0921 (ESM 2).

\section{Methods of Intervention}

The 36 retrieved studies covered six intervention schemes (Table 1). Among the single interventions, (1) three studies tested the passive distribution of the guideline or educational materials. $^{37,49,50}$ (2) Seven studies applied educational meetings, eventually including supplementary tools of the same methodological approach, e.g. distribution of materials applied on the meeting or e-learning sessions. ${ }^{34,40,44,47,54,57,58}$ (3) Seven studies used diverse single approaches including audit, ${ }^{25,31,43}$ motivational

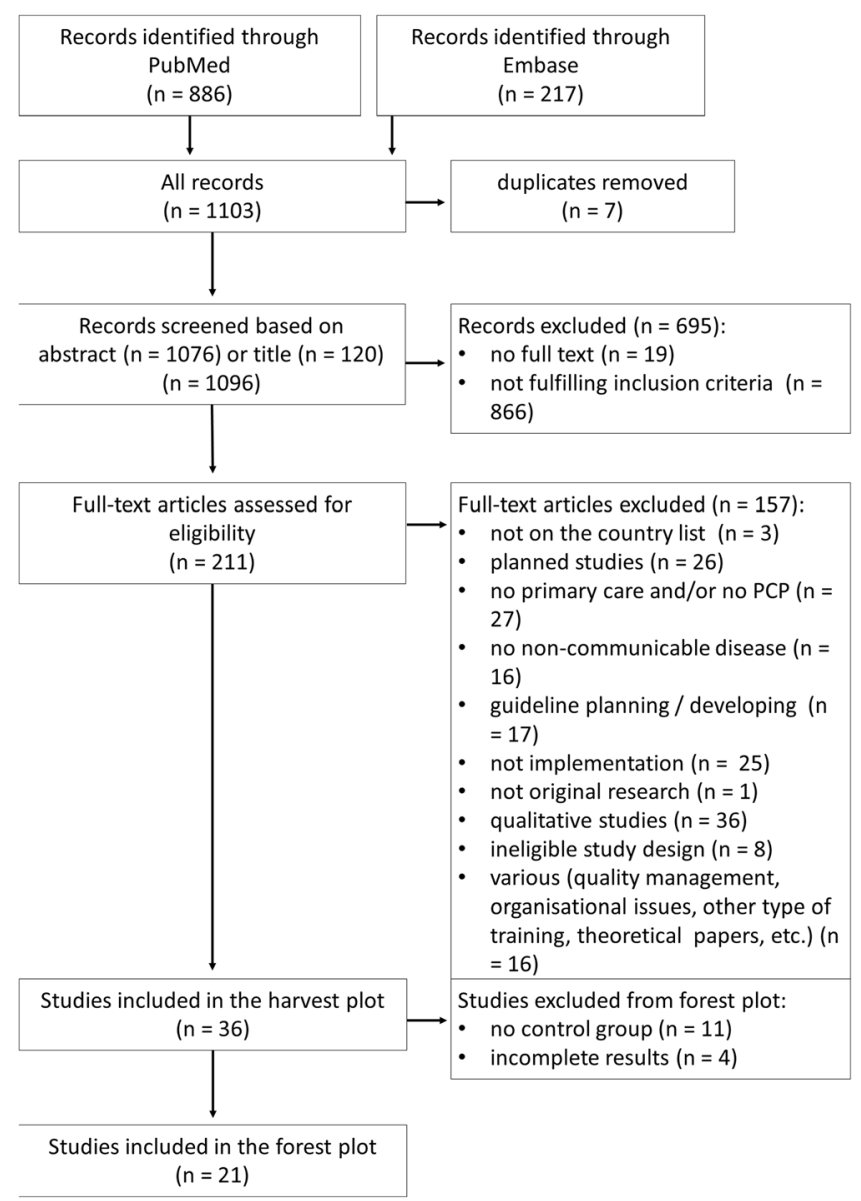

Figure 1 Flowchart of paper selection. 
Table 1 Characteristics of Selected Papers

\begin{tabular}{|c|c|c|c|c|c|c|c|}
\hline $\begin{array}{l}\text { First author, } \\
\text { year, country }\end{array}$ & $\begin{array}{l}\text { Targeted } \\
\text { guideline }\end{array}$ & Study design & $\begin{array}{l}\text { Included in } \\
\text { forest plot } \\
\text { (reasoning) }\end{array}$ & $\begin{array}{l}\text { Number of } \\
\text { PCPs in the } \\
\text { intervention } \\
\text { arm }\end{array}$ & $\begin{array}{l}\text { Applied intervention } \\
\text { scheme (theoretical } \\
\text { basis) }\end{array}$ & $\begin{array}{l}\text { Outcome } \\
\text { category } \\
\text { (number of } \\
\text { indicators) }\end{array}$ & $\begin{array}{l}\text { Quality } \\
\text { score }^{\dagger}\end{array}$ \\
\hline $\begin{array}{l}\text { Aakhus } \\
2016^{24} \text {; } \\
\text { Norway }\end{array}$ & Depression & CT & $\begin{array}{l}\text { Yes (main } \\
\text { outcome) }\end{array}$ & 51 & $\begin{array}{l}\text { outreach visit, } \\
\text { distribution nested in a } \\
\text { complex community } \\
\text { intervention }\end{array}$ & $\begin{array}{l}\text { Diagnostic } \\
\text { behaviour (5) } \\
\text { Outcome on } \\
\text { patient level } \\
\text { (4) }\end{array}$ & 2.5 \\
\hline $\begin{array}{l}\text { Anderson } \\
2014^{25} \text {; USA }\end{array}$ & $\begin{array}{l}\text { Chronic opioid } \\
\text { therapy }\end{array}$ & $\mathrm{BA}$ & $\begin{array}{l}\text { No (lack of } \\
\text { control group) }\end{array}$ & $\begin{array}{l}\text { Not reported } \\
\text { (community } \\
\text { health centre) }\end{array}$ & $\begin{array}{l}\text { audit (a dashboard to } \\
\text { serve as a central, } \\
\text { actionable data } \\
\text { repository to show } \\
\text { prescribing and } \\
\text { adherence rates) }\end{array}$ & $\begin{array}{l}\text { Diagnostic } \\
\text { behaviour (2) } \\
\text { Prescription } \\
\text { (1) } \\
\text { Counselling } \\
\text { (1) }\end{array}$ & 1.5 \\
\hline $\begin{array}{l}\text { Arts } 2017^{26} \text {; } \\
\text { the } \\
\text { Netherlands }\end{array}$ & Atrial fibrillation & $\mathrm{CT}$ & Yes & 13 & $\begin{array}{l}\text { reminder: clinical } \\
\text { decision support } \\
\text { systems integrated in } \\
\text { electronic health record }\end{array}$ & $\begin{array}{l}\text { Prescription } \\
\text { (1) }\end{array}$ & 1.75 \\
\hline $\begin{array}{l}\text { Bermejo et al. } \\
2009^{27} \text {; } \\
\text { Germany }\end{array}$ & Depression & CBA & $\begin{array}{l}\text { No (PCP results } \\
\text { not clearly } \\
\text { distinguishable) }\end{array}$ & 18 & $\begin{array}{l}\text { educational meeting as } \\
\text { continuous medical } \\
\text { education and } \\
\text { interdisciplinary } \\
\text { quality circles }(6 \text { times } \\
3 \mathrm{~h}) \text {; audit based on } \\
\text { weak point analysis }\end{array}$ & $\begin{array}{l}\text { Diagnostic } \\
\text { behaviour (1) } \\
\text { Counselling } \\
\text { (1) }\end{array}$ & 1.5 \\
\hline $\begin{array}{l}\text { Bonds et al. } \\
2009^{28} \text {; USA }\end{array}$ & Hypertension & CT & $\begin{array}{l}\text { Yes (main } \\
\text { outcomes) }\end{array}$ & 32 & $\begin{array}{l}\text { educational meeting, } \\
\text { followed by outreach } \\
\text { visits in every } 6 \\
\text { months, extended by } \\
\text { distribution of written } \\
\text { education material and } \\
\text { of patients tools; audit } \\
\text { provided by external } \\
\text { auditor firm }\end{array}$ & $\begin{array}{l}\text { Prescription } \\
\text { (3) } \\
\text { Counselling } \\
\text { (1) } \\
\text { Outcome on } \\
\text { patient level } \\
\text { (4) }\end{array}$ & 2.75 \\
\hline $\begin{array}{l}\text { Braun et al. } \\
2011^{29} \text {; } \\
\text { Germany }\end{array}$ & $\begin{array}{l}\text { Chronic heart } \\
\text { failure guidelines }\end{array}$ & $\mathrm{BA}$ & $\begin{array}{l}\text { No (lack of } \\
\text { control group) }\end{array}$ & 23 & $\begin{array}{l}\text { educational meeting as } \\
\text { introductory seminar, } \\
\text { followed by installing } \\
\text { a reminder on the } \\
\text { praxis computer with } \\
\text { pop-up window of the } \\
\text { guideline essentials }\end{array}$ & $\begin{array}{l}\text { Prescription } \\
\text { (4) }\end{array}$ & 1.5 \\
\hline $\begin{array}{l}\text { Chen } 2016^{30} \text {; } \\
\text { USA }\end{array}$ & $\begin{array}{l}\text { Chronic opioid } \\
\text { therapy }\end{array}$ & BA & $\begin{array}{l}\text { No (lack of } \\
\text { control group) }\end{array}$ & Not reported & $\begin{array}{l}\text { educational meeting, } \\
\text { dissemination, } \\
\text { networking }\end{array}$ & $\begin{array}{l}\text { Diagnostic } \\
\text { behaviour (4) } \\
\text { Prescription } \\
(2)\end{array}$ & 1.5 \\
\hline $\begin{array}{l}\text { Dormuth } \\
\text { et al. 2012 } \\
\text { Canada }\end{array}$ & Statin prescription & CT & Yes & 1394 & $\begin{array}{l}\text { audit: feedback } \\
\text { provided on } \\
\text { prescription }\end{array}$ & $\begin{array}{l}\text { Prescription } \\
\text { (2) }\end{array}$ & 2.25 \\
\hline $\begin{array}{l}\text { Ennis, } \\
2015^{32} \text {; USA }\end{array}$ & $\begin{array}{l}\text { Chronic kidney } \\
\text { disease }\end{array}$ & CT & Yes & Not reported & $\begin{array}{l}\text { reminder: automated } \\
\text { laboratory-based clini- } \\
\text { cal decision support } \\
\text { system }\end{array}$ & $\begin{array}{l}\text { Diagnostic } \\
\text { behaviour } \\
(10) \\
\text { Outcome on } \\
\text { patient level } \\
\text { (9) }\end{array}$ & 1.5 \\
\hline $\begin{array}{l}\text { Franx et al. } \\
2014^{33} \text {; } \\
\text { Netherlands }\end{array}$ & Depression & CBA & Yes & 20 & $\begin{array}{l}2 \text { days educational } \\
\text { meeting and additional } \\
\text { conference days; } \\
\text { followed by virtual } \\
\text { networking of } \\
\text { multidisciplinary teams } \\
\text { for online discussions } \\
\text { exchanging of best } \\
\text { practices, supported by } \\
\text { a toolkit of instruments } \\
\text { as treatment protocols } \\
\text { or links to relevant } \\
\text { sites }\end{array}$ & $\begin{array}{l}\text { Prescription } \\
(1) \\
\text { Diagnostic } \\
\text { behaviour (1) }\end{array}$ & 2 \\
\hline
\end{tabular}

interview, ${ }^{53}$ reminder $^{26,32}$ and patient-mediated intervention. ${ }^{41}$ Multifaceted interventions mainly applied a combination of two methods, one of them a kind of training approach, extended by a second methodologically different method. From these, (4) the combination of educational meeting and audit/feedback was the most frequent with four studies. ${ }^{27,46,52,56}$ (5) Seven studies 
Table 1. (continued)

\begin{tabular}{|c|c|c|c|c|c|c|c|}
\hline $\begin{array}{l}\text { First author, } \\
\text { year, country }\end{array}$ & $\begin{array}{l}\text { Targeted } \\
\text { guideline }\end{array}$ & Study design & $\begin{array}{l}\text { Included in } \\
\text { forest plot } \\
\text { (reasoning) }\end{array}$ & $\begin{array}{l}\text { Number of } \\
\text { PCPs in the } \\
\text { intervention } \\
\text { arm }\end{array}$ & $\begin{array}{l}\text { Applied intervention } \\
\text { scheme (theoretical } \\
\text { basis) }\end{array}$ & $\begin{array}{l}\text { Outcome } \\
\text { category } \\
\text { (number of } \\
\text { indicators) }\end{array}$ & $\begin{array}{l}\text { Quality } \\
\text { score }^{\dagger}\end{array}$ \\
\hline $\begin{array}{l}\text { French et al. } \\
2013^{34} ; \\
\text { Australia }\end{array}$ & $\begin{array}{l}\text { Acute low back } \\
\text { pain }\end{array}$ & $\mathrm{CT}$ & $\begin{array}{l}\text { Yes (excluding } \\
\text { simulated } \\
\text { diagnostic } \\
\text { behaviour) }\end{array}$ & 45 & $\begin{array}{l}\text { educational meeting } \\
\text { combining didactic } \\
\text { lectures and small } \\
\text { group discussions; } \\
\text { supported by providing } \\
\text { the educational } \\
\text { material on DVD } \\
\text { (Theoretical Domains } \\
\text { Framework and the } \\
\text { Theory of Planned } \\
\text { Behaviour) }\end{array}$ & $\begin{array}{l}\text { Knowledge } \\
\text { transfer (4) } \\
\text { Diagnostic } \\
\text { behaviour (3) }\end{array}$ & 2.5 \\
\hline $\begin{array}{l}\text { Grunfeld et al. } \\
2013^{35} \\
\text { Canada }\end{array}$ & $\begin{array}{l}\text { Chronic disease } \\
\text { prevention and } \\
\text { screening }\end{array}$ & CBA & $\begin{array}{l}\text { Yes (main } \\
\text { outcome) }\end{array}$ & 4 & $\begin{array}{l}\text { outreach visit extended } \\
\text { by practice facilitation } \\
\text { and audit (the patient- } \\
\text { targeted arm of inter- } \\
\text { vention was skipped in } \\
\text { our analysis) }\end{array}$ & $\begin{array}{l}\text { Diagnostic } \\
\text { behaviour } \\
(28)\end{array}$ & 2.75 \\
\hline $\begin{array}{l}\text { Harris } 2015^{36} \\
\text { Australia }\end{array}$ & $\begin{array}{l}\text { Prevention of } \\
\text { cardiovascular } \\
\text { disease and type } 2 \\
\text { diabetes }\end{array}$ & $\mathrm{CT}$ & Yes & 42 & $\begin{array}{l}\text { educational meeting, } \\
\text { audit and feedback, } \\
\text { outreach visit (the } \\
\text { patient-targeted educa- } \\
\text { tion and referral infor- } \\
\text { mation was skipped in } \\
\text { our analysis) }\end{array}$ & $\begin{array}{l}\text { Diagnostic } \\
\text { behaviour ( } 8)\end{array}$ & 2.25 \\
\hline $\begin{array}{l}\text { Heppe et al. } \\
2012^{37} \text {; USA }\end{array}$ & $\begin{array}{l}\text { Cardiovascular risk } \\
\text { assessment }\end{array}$ & BA & $\begin{array}{l}\text { No (lack of } \\
\text { control group) }\end{array}$ & 234 & $\begin{array}{l}\text { distribution of } \\
\text { guideline via mail }\end{array}$ & $\begin{array}{l}\text { Knowledge } \\
\text { transfer (1) }\end{array}$ & 1.5 \\
\hline $\begin{array}{l}\text { Hunt et al. } \\
2009^{38} \text {; USA }\end{array}$ & Diabetes & BA & $\begin{array}{l}\text { No (lack of } \\
\text { control group) }\end{array}$ & 71 & $\begin{array}{l}\text { educational meeting } \\
\text { providing guided tours } \\
\text { of system navigation } \\
\text { and functionality; } \\
\text { integrated point-of- } \\
\text { care reminder and } \\
\text { web-based tools; } \\
\text { monthly audit }\end{array}$ & $\begin{array}{l}\text { Diagnostic } \\
\text { behaviour (6) } \\
\text { Prescription } \\
\text { (4) } \\
\text { Outcome on } \\
\text { patient level } \\
(5)\end{array}$ & 1.5 \\
\hline $\begin{array}{l}\text { Ioannidis et el } \\
2009^{39} \\
\text { Canada }\end{array}$ & Osteoporosis & BA & $\begin{array}{l}\text { No (lack of } \\
\text { control group) }\end{array}$ & 340 & $\begin{array}{l}\text { multifaceted scheme of } \\
\text { educational meeting, } \\
\text { reminder, audit, } \\
\text { opinion leaders, } \\
\text { financial } \\
\text { reimbursement, } \\
\text { patient-medicated in- } \\
\text { terventions and distri- } \\
\text { bution of educational } \\
\text { material }\end{array}$ & $\begin{array}{l}\text { Prescription } \\
\text { (3) }\end{array}$ & 1.5 \\
\hline $\begin{array}{l}\text { Kiessling } \\
\text { et al. } 2011^{40} \text {; } \\
\text { Sweden }\end{array}$ & $\begin{array}{l}\text { Lipid lowering in } \\
\text { patients with } \\
\text { coronary heart } \\
\text { disease }\end{array}$ & $\mathrm{CT}$ & Yes & 26 & $\begin{array}{l}\text { educational meeting } \\
\text { for case discussion in } \\
\text { small groups }\end{array}$ & $\begin{array}{l}\text { Outcome on } \\
\text { patient level } \\
\text { (1) }\end{array}$ & 2.75 \\
\hline $\begin{array}{l}\text { Licskai et al. } \\
2012^{41} \\
\text { Canada }\end{array}$ & Asthma & BA & $\begin{array}{l}\text { No (lack of } \\
\text { control group) }\end{array}$ & 60 & $\begin{array}{l}\text { patient-mediated } \\
\text { intervention involving } \\
\text { an asthma educator } \\
\text { (knowledge translation } \\
\text { framework by the } \\
\text { Canadian Institutes of } \\
\text { Health Research) }\end{array}$ & $\begin{array}{l}\text { Prescription } \\
\text { (4) } \\
\text { Outcome on } \\
\text { patient level } \\
(6) \text { : in the } \\
\text { harvest plot } \\
\text { only }\end{array}$ & 1.75 \\
\hline $\begin{array}{l}\text { Liddy } 2015^{42} \\
\text { Canada }\end{array}$ & $\begin{array}{l}\text { Secondary } \\
\text { prevention of heart } \\
\text { disease, stroke, } \\
\text { peripheral vascular } \\
\text { disease, renal } \\
\text { disease and } \\
\text { diabetes }\end{array}$ & $\begin{array}{l}\text { Stepped } \\
\text { wedge cluster } \\
\text { randomized } \\
\text { trial }\end{array}$ & $\begin{array}{l}\text { No (data } \\
\text { presentation) }\end{array}$ & 182 & $\begin{array}{l}\text { outreach visit, audit } \\
\text { and feedback, } \\
\text { networking }\end{array}$ & $\begin{array}{l}\text { Diagnostic } \\
\text { behaviour (1) }\end{array}$ & 2.75 \\
\hline $\begin{array}{l}\text { Mahlknecht, } \\
2016^{43} \\
\text { Austria }\end{array}$ & $\begin{array}{l}\text { Several chronic } \\
\text { disease guidelines }\end{array}$ & BA & $\begin{array}{l}\text { No (lack of } \\
\text { control group) }\end{array}$ & 20 & $\begin{array}{l}\text { audit: benchmarking } \\
\text { of } 43 \text { consensual } \\
\text { quality indicators, } \\
\text { networking in quality } \\
\text { circles }\end{array}$ & $\begin{array}{l}\text { Diagnostic } \\
\text { behaviour } \\
\text { (43) }\end{array}$ & 2 \\
\hline $\begin{array}{l}\text { Mallen } \\
2017^{44} \text {; UK }\end{array}$ & Osteoarthritis & $\mathrm{CT}$ & Yes & 20 & educational meeting & $\begin{array}{l}\text { Outcome on } \\
\text { patient level } \\
\text { (1) }\end{array}$ & 2.5 \\
\hline
\end{tabular}


Table 1. (continued)

\begin{tabular}{llllll}
\hline $\begin{array}{l}\text { First author, } \\
\text { year, country }\end{array}$ & $\begin{array}{l}\text { Targeted } \\
\text { guideline }\end{array}$ & Study design & $\begin{array}{l}\text { Included in } \\
\text { forest plot } \\
\text { (reasoning) }\end{array}$ & $\begin{array}{l}\text { Number of } \\
\text { PCPs in the } \\
\text { intervention } \\
\text { arm }\end{array}$ & $\begin{array}{l}\text { Applied intervention } \\
\text { scheme (theoretical } \\
\text { basis) }\end{array}$ \\
\hline $\begin{array}{l}\text { Mold } 2014^{45} ; \\
\text { USA }\end{array}$ & Kidney disease & BA & $\begin{array}{l}\text { No (lack of } \\
\text { control group) }\end{array}$ & 58 & audit, outreach visit in \\
wave II
\end{tabular}

$\begin{array}{ll}\begin{array}{l}\text { Outcome } \\ \text { category } \\ \text { (number of } \\ \text { indicators) }\end{array} & \begin{array}{l}\text { Quality } \\ \text { score }^{\dagger}\end{array} \\ & \end{array}$

Diagnostic

behaviour (2)

Prescription

(2)

Outcome on

patient level

(4)

outcome)

18

et al. $2009^{46}$; heart failure

Germany

$\begin{array}{ll}\text { CT } & \text { Yes (main } \\ \text { outcome) }\end{array}$

CBA

CT

Screening for depression

Yes
Secher et al. Resuscitation

$2012^{49}$;

Denmark

Shah et al. Cardiovascular

$2014^{50}$;

Canada

disease
CT

Yes (main

outcome)

Yes (main

outcome)

12

depression

$2015^{51}$; the

Netherlands

Sipilä et al. Hypertension

$2011^{52}$;

Finland

$\begin{array}{ll}\begin{array}{l}\text { Skoglund } \\ \text { et al. 2013 } \\ \text { Sweden }\end{array} & \text { Hypertension } \\ \begin{array}{l}\text { Soler et al. } \\ 2010^{54} \text {; Spain }\end{array} & \begin{array}{l}\text { Chronic } \\ \text { obstructive } \\ \text { pulmonary disease }\end{array} \\ \begin{array}{l}\text { Tahvonen } \\ 2016^{55} ;\end{array} & \text { Spine radiography } \\ \text { Finland } & \end{array}$

educational meeting

with multifaceted

approaches including

structured case

discussions on real

patients,

communication

training and practice

organisation; audit

Not reported

presentation)

30

1 day educational

meeting by a psychiatrist

in small group; monthly

reminders by email and

feedback from the PCP

on adherence and

feasibility

distribution of

guideline in direct

mail, the cover letter

outlining changes;

supported by a poster

distribution of a

toolbox: an

introductory letter; a

synopsis of the key

guideline elements

pertaining to

cardiovascular disease

risk; a laminated card

with an algorithm for

risk assessment and

screening for

cardiovascular disease;

and a pad of tear-off

sheets for patients with

risk self-assessment

tool and a list of risk

reduction strategies

educational meetings

in both arms;

intervention only:

motivational interview,

networking targeted to

identified barriers

16 educational

meetings (lectures,

workshops, patient

cases and role

modelling) and 12

distance learning tasks

in 2 years; audit

Providing evidence-

based drug information

with motivational in-

terview technique

educational meeting

by pulmonologists and

training on spirometry

educational meeting,

distribution
Diagnostic

behaviour (2)

Prescription

(4)

Outcome on

patient level

(6)

Knowledge transfer (1)

Diagnostic behaviour (1)

Prescription

(1)

Knowledge

transfer (9)

Outcome on

atient level

(15): in the

harvest plot

only

Diagnostic

behaviour (5):

in the harvest

plot only

Prescription

(10): in the

harvest plot

only

Diagnostic

behaviour (2)

Prescription

(1)

Counselling

(1)

Prescription

(16)

2

5

Prescription

Diagnostic

behaviour (6)

Prescription

(9)

Diagnostic

behaviour (1) 
Table 1. (continued)

\begin{tabular}{|c|c|c|c|c|c|c|c|}
\hline $\begin{array}{l}\text { First author, } \\
\text { year, country }\end{array}$ & $\begin{array}{l}\text { Targeted } \\
\text { guideline }\end{array}$ & Study design & $\begin{array}{l}\text { Included in } \\
\text { forest plot } \\
\text { (reasoning) }\end{array}$ & $\begin{array}{l}\text { Number of } \\
\text { PCPs in the } \\
\text { intervention } \\
\text { arm }\end{array}$ & $\begin{array}{l}\text { Applied intervention } \\
\text { scheme (theoretical } \\
\text { basis) }\end{array}$ & $\begin{array}{l}\text { Outcome } \\
\text { category } \\
\text { (number of } \\
\text { indicators) }\end{array}$ & $\begin{array}{l}\text { Quality } \\
\text { score }^{\dagger}\end{array}$ \\
\hline $\begin{array}{l}\text { Valles- } \\
\text { Fernandez et } \\
\text { el } 2012^{56} \text {; } \\
\text { Spain }\end{array}$ & Hypertension & $\mathrm{CT}$ & Yes & 430 & $\begin{array}{l}8 \text { educational meetings } \\
\text { in } 2 \text { years, supported } \\
\text { by posters and leaflets, } \\
\text { audit in every } 6 \\
\text { months }\end{array}$ & $\begin{array}{l}\text { Diagnostic } \\
\text { behaviour (1) } \\
\text { Outcome on } \\
\text { patient level } \\
\text { (1) }\end{array}$ & 2 \\
\hline $\begin{array}{l}\text { Verbiest } \\
2014^{57} \text {; the } \\
\text { Netherlands }\end{array}$ & Smoking cessation & CT & $\begin{array}{l}\text { Yes (main } \\
\text { outcome) }\end{array}$ & 25 & $\begin{array}{l}\text { educational meeting, } \\
\text { action planning }\end{array}$ & $\begin{array}{l}\text { Counselling } \\
\text { (1) }\end{array}$ & 1.75 \\
\hline $\begin{array}{l}\text { Vidal Pardo } \\
\text { et al. } 2013^{58} \text {; } \\
\text { Spain }\end{array}$ & Diabetes & CBA & Yes & 58 & $\begin{array}{l}\text { educational meeting as } \\
3 \text { 90-min workshops; } \\
\text { supported by providing } \\
\text { the educational materi- } \\
\text { al and by other online } \\
\text { teaching resources as } \\
\text { forum, tutorial and } \\
\text { case resolution }\end{array}$ & $\begin{array}{l}\text { Diagnostic } \\
\text { behaviour } \\
\text { (10) }\end{array}$ & 2.5 \\
\hline $\begin{array}{l}\text { Wentworth } \\
\text { et al. } 2011^{59} \text {; } \\
\text { USA }\end{array}$ & Kidney disease & BA & $\begin{array}{l}\text { No (lack of } \\
\text { control group) }\end{array}$ & 3 & $\begin{array}{l}\text { distribution of } \\
\text { educational material } \\
\text { (1-page quick } \\
\text { reference and patient } \\
\text { guide); outreach visit } \\
\text { as academic detailing } \\
\text { and practice } \\
\text { enhancement assistants } \\
\text { implementing a } \\
\text { computer-guided, } \\
\text { point-of-care decision- } \\
\text { support system, pro- } \\
\text { viding reminder during } \\
1 \text { year (RE-AIM } \\
\text { framework) }\end{array}$ & $\begin{array}{l}\text { Diagnostic } \\
\text { behaviour (8) } \\
\text { Prescription } \\
\text { (4) } \\
\text { Outcome on } \\
\text { patient level } \\
\text { (4) }\end{array}$ & 2 \\
\hline
\end{tabular}

CT controlled trial; (C)BA (controlled) before-after study

${ }^{\top}$ Mean of the rating of four criteria (random allocation to the interventions, blinding the outcome assessors, completeness of the outcome data, avoiding selective reporting, each rated as no problem (3 points); probably no problem (2 points); and no info or a reported problem (1 point)

applied educational meetings supported by diverse additional methods such as reminders, ${ }^{29,48}$ networking ${ }^{33}$ or distribution ${ }^{55}$; or performed the educational component in the form of an outreach visit, extended by distribution ${ }^{24}$ or audit. ${ }^{42,45}$ (6) Eight studies used a multifaceted approach, combining more than two methods: distribution of materials, outreach visit and reminders ${ }^{59}$; educational meetings, outreach visit and audit ${ }^{28,36}$; outreach visit, audit and practice facilitation ${ }^{35}$; educational meetings, audit and reminders ${ }^{38}$; educational meetings, dissemination and networking $^{30}$; educational meetings, motivational interview and networking $^{51}$; educational meetings, reminders, audit and feedback, opinion leaders, financial reimbursement, patient-medicated interventions and distribution of educational material. ${ }^{39}$

Three studies reported explicitly relying on a theory with regard to the tested intervention: the Theoretical Domains Framework and the Theory of Planned Behaviour, ${ }^{34}$ the knowledge translation framework by the Canadian Institutes of Health Research ${ }^{41}$ and the RE-AIM framework. ${ }^{59}$

\section{Indicators}

The range of the number of indicators within a study was one to 43 (Table 1); in total, 303 outcome indicators were reported in the 36 studies. Eighteen studies applied outcome indicators belonging to one single outcome category, i.e. either prescription, diagnostic behaviour, counselling, knowledge or patient-level outcome of care; the other 18 studies covered up to three outcome categories in individual combinations (Table 1). In total, 62 groups of outcome indicators were reported by the 36 studies, referring to knowledge transfer (in four studies, with a range of one to nine individual indicators), diagnostic behaviour (in 22 studies, with a range of one to 43 individual indicators), prescription (in 19 studies, with a range of one to 16 individual indicators), counselling activity (in five studies, with each one indicator) and patient-level outcomes (in 12 studies, with a range of one to 15 individual indicators). With regard to effectiveness, 27 outcome groups showed no effect, 14 a moderate and 21 a strong effect.

\section{Impact of Intervention}

The analysis according to the intervention schemes was performed both by a harvest plot (Fig. 2) and forest plot (ESM 3) demonstrating that more complex methods are not necessarily more effective. Diverse single intervention methods such as audit, reminder, motivational 


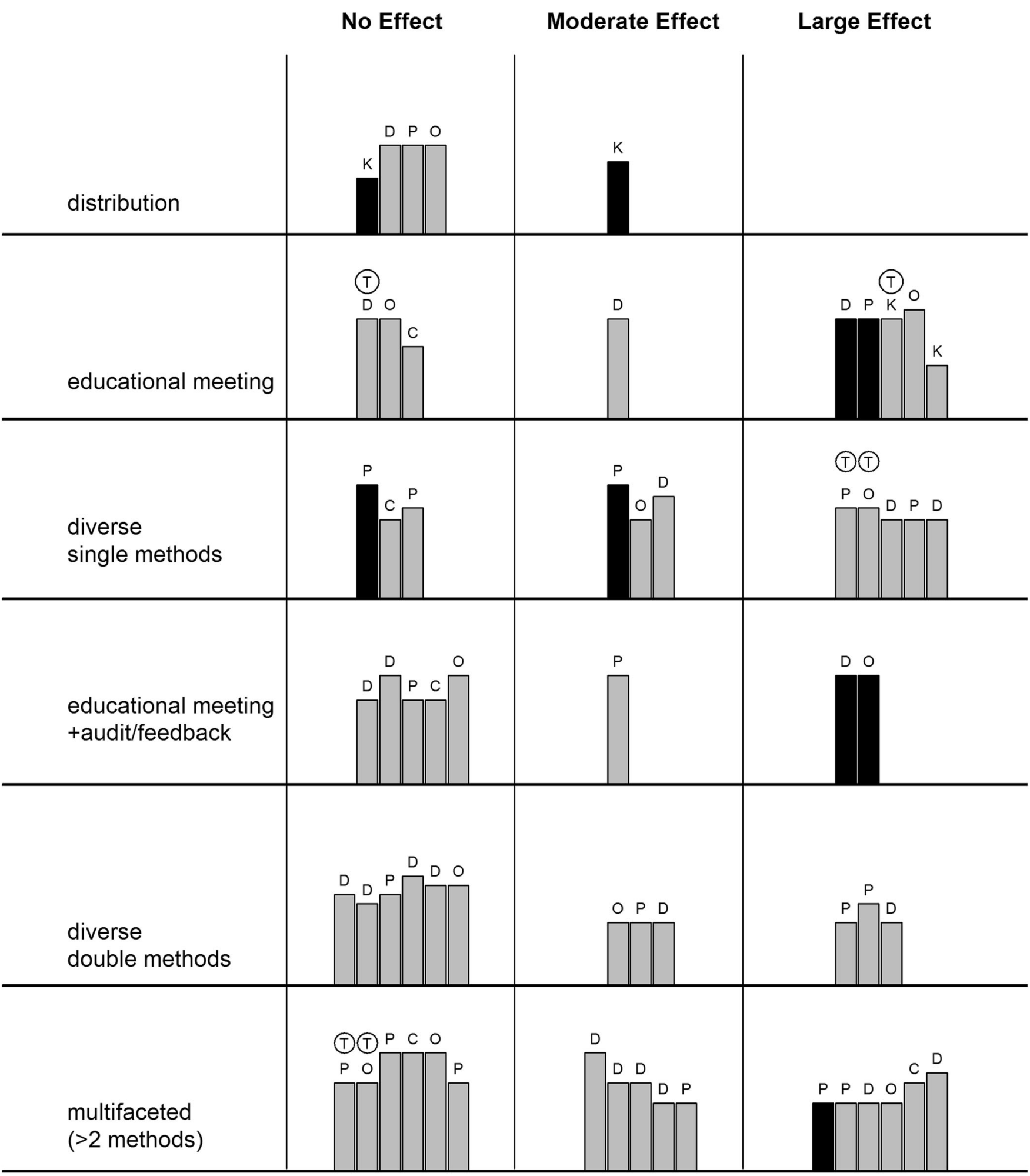

Figure 2 Impact of intervention (harvest plot). Height of the bar: quality of the paper. Colour of the bar: number of PCPs in the intervention (grey < 100, black $\geq 100$ ). Category of the outcome: D: diagnostic behaviour, P: prescription, C: counselling, K: knowledge level, O: outcome of care on patient level, $\mathrm{T}$ : theory-based intervention.

interview or patient-mediated intervention showed the largest effect $(73 \%$ of outcome indicators being effective; $0.48[0.38,0.58])$ covering all outcome categories except counselling. A similar rate of success was observable for educational meetings with $67 \%$ of indicators being effective $(0.18[0.06,0.31])$ and multifaceted 
interventions combining more than two methods (with $65 \%$ of indicators being effective; $0.11[0.01,0.20]$ ).

In contrast, the least effective method was the passive distribution of materials not implying any organised activity from the target group, showing a moderate effect only in the knowledge transfer category.

The effectiveness of multifaceted interventions applying two methods lay in between the two above approaches. Combining the educational meeting with audit almost halved the effectiveness (38\% of the indicators showing any effect) compared to the single educational meeting, while the effectiveness of other two-method combinations was better but still not achieving the result of single-method active interventions.

The effectiveness of all single interventions even including the distribution of materials was larger $(0.27[0.17,0.38])$ (ESM 4) than the effectiveness of all multifaceted interventions applying more than one method $(0.13[0.06,0.19])$ (ESM $5)$.

The random effects model of quantitative outcomes based on 73 indicators of 21 studies covering five outcome categories shown in the forest plot (Fig. 3) resulted in a moderate overall positive summary effect measure $(0.22[0.15,0.29])$.

Theory-based interventions (Fig. 2) did not show better performance, compared to those without explicitly referring to theory.

Analysing the effectiveness according to the outcome categories, knowledge transfer showed the largest improvement $(0.39[0.05,0.73])$. For the outcome category of diagnostic behaviour, a moderate positive effect was detected $(0.22[0.14$, $0.31])$. Prescription showed no significant improvement $(0.11$ $[-0.01,0.24])$, likewise the patient-based outcomes $(0.14$ [$0.02,0.30])$. The analysis of the counselling activity was based on two indicators where the achievement of a single study contributed to the improvement $(0.30[0.14,0.45])$, while in the harvest plot, only one of the five indicators showed an improvement.

\section{DISCUSSION}

This study demonstrated that, among a wide span of interventions targeted at guideline implementation in the primary care setting, single-component interventions were equally effective as complex multifaceted intervention schemes in improving process of care and outcome of care.

When promoting a guideline, it seems obvious that educational material such as leaflets, folders or posters should be distributed. However, our analysis could show that passively receiving educational materials was least effective. Benefits of traditional printed educational materials are modest and short term. ${ }^{12}$ Arguably, the modest effect of passive distribution of educational material could be improved, e.g. by wisely choosing the channel and method of delivery ${ }^{13}$ or by the design of the material. ${ }^{60}$
An intervention should not stop at this step: also educational meetings, when remaining passive and didactic, could not improve the practice, regardless of whether it improved knowledge or not. ${ }^{61}$ In contrast, approaches implying active participation in the educational process have been reported as a key factor for success. ${ }^{62}$ This aspect is reflected when looking at the details explaining the difference between the high effectiveness of educational meetings found in our study and the controversial opinions regarding the effectiveness of educational meetings reported in the literature. ${ }^{7,63}$ Interactive education, preferably combined with supportive measures, ${ }^{61}$ was found to be superior to didactic and passive education, ${ }^{13}$ being able to improve either the process of care performed by the PCPs or the health outcomes of the patients. ${ }^{13,61}$ However, the effect sizes are typically small, ${ }^{12}$ because education alone has a limited effect on modifying complex behaviours. ${ }^{61}$

These above methods represent the two ends of the spectrum of the effectiveness detected in this study. In between, several single-intervention methods and their combinations may demonstrate effectiveness or lack of it, depending on the adjustment to the current circumstances. The generally low impact of audit and feedback ${ }^{12,13,64}$ can be improved by methodological refinement ${ }^{64}$ like choosing a respected authority as a source of providing repeated feedback in both verbal and written forms, or applying management tools of clear target setting and an action plan for achieving them. Reminders have a moderate effect on guideline implementation $^{12,65,66}$ either as a single approach or in combination with educational materials or meetings as part of a multifaceted intervention. ${ }^{12}$ No consistent characteristics could be identified to increase their effect; ${ }^{65}$ however, some studies found a better performance of computer-based reminders compared to paper-based. ${ }^{13}$ In our analysis, only one study ${ }^{41}$ applied patient-mediated intervention with convincing effect on prescription and also on outcomes on patient level. Literature ${ }^{12}$ also suggests moderate to large improvement.

In our study, multifaceted interventions did not demonstrate a direct relationship between the number of intervention components and effect size, the same result confirmed by literature. ${ }^{12,67}$ The benefit of multifaceted interventions is ambiguous: some reviews did not support the commonly held assumption that multifaceted interventions are more effective than single-component interventions, ${ }^{12}$ while others favoured the multifaceted approach. ${ }^{13,62}$

These results suggest possibilities for further improvement. Our search hits covered an almost complete range of professional interventions, but it is equally important what was not covered: no example for organisational interventions could be identified. The literature confirms that this approach is sparse $^{12,13}$ : most of the interventions focus on the providers' behaviour on individual level in spite of the fact that organisational interventions could give a powerful support for behaviour change ${ }^{63}$ or limit its required extent. ${ }^{68}$ Addressing the context comprehensively to increase the effectiveness could be supported by relying on theoretical frameworks. ${ }^{69}$ 


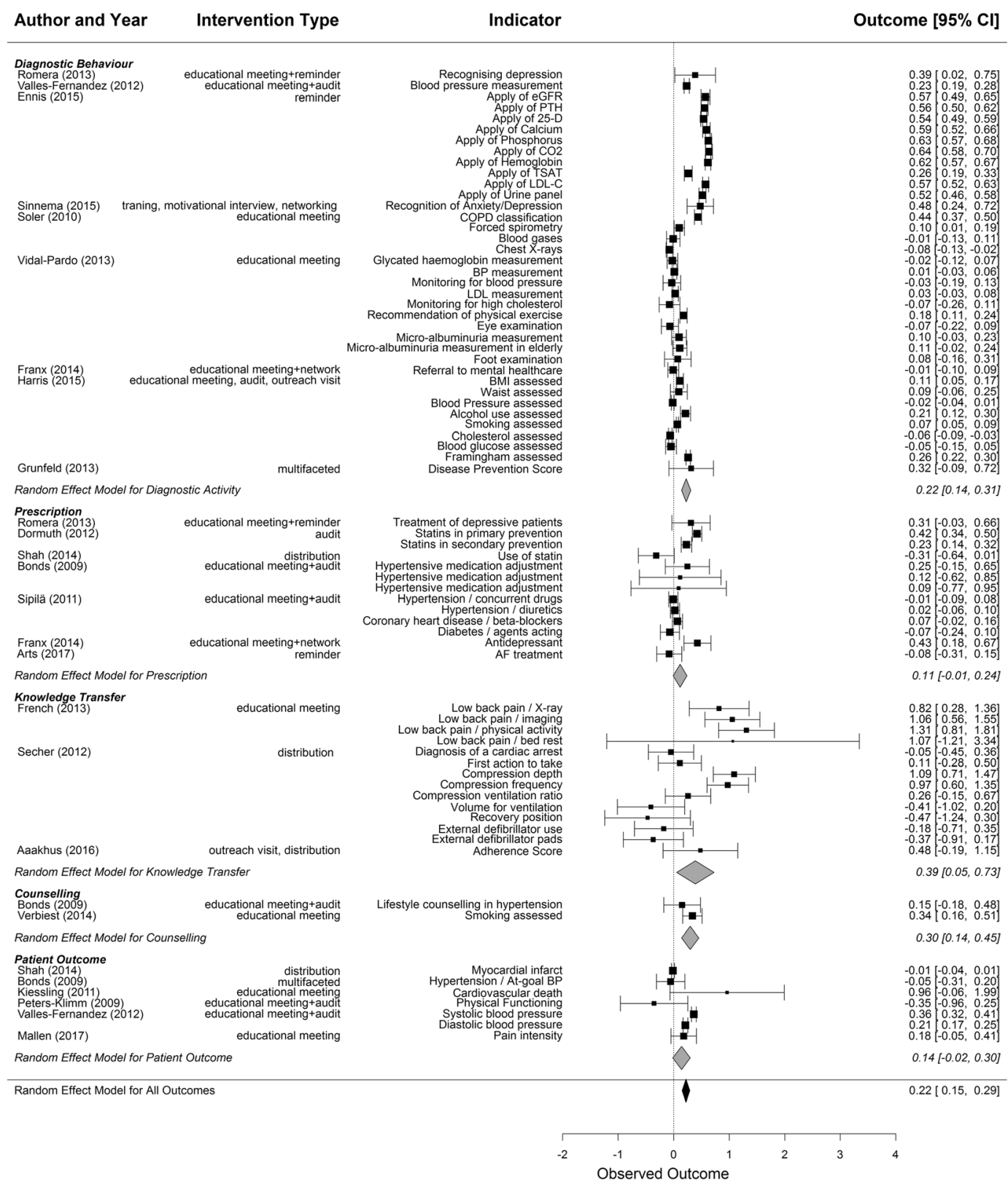

Figure 3 Impact of intervention (forest plot) separated for outcome categories. The summary measure for each outcome category is shown at the end of each category; the summary measure over all studies at the bottom. Size of box proportional to log of sample size.

However, with respect to the small sample size in our review of studies utilising a theory, no definite conclusion could be drawn regarding the performance of a theory-based approach. The literature also reports ${ }^{70,71}$ on the small number of studies applying a theoretical background and encourages its use tailored to the setting and to the aim of intervention. ${ }^{70}$ Economic evaluation of guideline implementation interventions is rarely reported, ${ }^{12,13}$ our hits demonstrating no exception: three studies $^{31,35,41}$ presented cost-benefit or cost-effectiveness information. This indicates that research should focus on adding this essential aspect in favour of comprehensive comparability. ${ }^{72}$ Finally, including the above aspects already in the guidelines $^{73}$ and addressing the needs of the providers and settings ${ }^{74}$ as well as those of the patients ${ }^{75}$ could represent a powerful tool for increasing implementation effectiveness.

\section{LIMITATIONS}

Among several interventions targeting the PCP, our search strategy was directed to the studies which explicitly stated guideline implementation in the title or abstract. Search was performed in two major literature databases; however, relevant studies still may have been missed. Applying the UNSD categorisation for the selection of the developed countries may not cover all aspects of interest regarding the developmental level of the health care systems.

The diverse quality of studies was indicated in the analysis. The selected studies were heterogeneous with regard to or have not reported in-depth the non-clinical factors potentially influencing adherence to the guidelines of evidence-based practice; e.g. patient-related factors such as socioeconomic 
status, attitude, or preferences; physician's personal characteristics, qualification, or work overload; and practice-related factors such as availability of resources or policies and reimbursement model. ${ }^{76}$

To account for the heterogeneity of the included studies regarding outcomes, study design and settings, we chose a random effects model and all effect measures were transformed to a similar scale. Still, the results should be interpreted with care and we utilised the forest plot rather as a tool to give an overview on the different effects, rather than to draw conclusions due to the summary measures. According to our best knowledge, applying forest plot and harvest plot on the same dataset to display and compare the information extracted by two means of synthesis of different level is a novelty.

\section{CONCLUSION}

Our systematic review demonstrated that all elements of the PCPs' activities can be successfully improved by intervention; however, the most effective method cannot be simply and consequently linked with the category of the targeted outcome. The best result could be detected when knowledge, a basic prerequisite for change, was provided by an interactive method, and the intervention scheme addressed the detected needs of the involved actors and the barriers of the setting. Organising a change from outside requires a deliberate balance between addressing the detected needs and minimising the load on the target group, resulting in an intervention scheme as complex as required but as simple as possible.

Corresponding Author: Eva Kovacs, Dr. med. PhD; Institute for Medical Information Processing, Biometrics and Epidemiology Ludwig-Maximilians-Universität, Munich, Germany (e-mail: Eva. Kovacs@med.uni-muenchen.de).

Funding Information This study was funded by the Federal Ministry of Education and Research, Germany (BMBF FKZ O1EO1401).

\section{Compliance with Ethical Standards:}

Conflict of Interest: The authors declare that they do not have a conflict of interest.

\section{REFERENCES}

1. Lau R, Stevenson F, Ong BN, Dziedzic K, Treweek S, Eldridge S, et al. Achieving change in primary care-causes of the evidence to practice gap: systematic reviews of reviews. Implement Sci 2016;11:40. https://doi. org/10.1186/s13012-016-0396-4

2. Bernstein AB, Hing $\mathbf{E}$, Burt $\mathbf{C W}$, Hall MJ. Trend data on medical encounters: tracking a moving target. Health Aff (Project Hope) 2001;20(2):58-72.

3. Starfield B, Shi L, Macinko J. Contribution of primary care to health systems and health. Milbank Q 2005;83(3):457-502. https://doi.org/10. $1111 /$ j.1468-0009.2005.00409.x

4. Gibson OR, Segal L, McDermott RA. A systematic review of evidence on the association between hospitalisation for chronic disease related ambulatory care sensitive conditions and primary health care resourcing. BMC Health Serv Res 2013;13:336. https://doi.org/10.1186/1472-6963-13-336

5. Fernandez A, Sturmberg J, Lukersmith S, Madden R, Torkfar G, Colagiuri R, et al. Evidence-based medicine: is it a bridge too far? Health Res Policy Syst 2015; 13:66. https://doi.org/10.1186/s12961-015-0057-0

6. Saarni SI, Gylling HA. Evidence based medicine guidelines: a solution to rationing or politics disguised as science? J Med Ethics 2004;30(2):171-5.

7. Brusamento S, Legido-guigley H, Panteli D, Turk E, Knai C, Saliba V, et al. Assessing the effectiveness of strategies to implement clinical guidelines for the management of chronic diseases at primary care level in EU Member States: a systematic review. Health Policy 2012;107(23): 168-83. https://doi.org/10.1016/j.healthpol.2012.08.005

8. Legido-Quigley H, Panteli D, Brusamento S, Knai C, Saliba V, Turk E, et al. Clinical guidelines in the European Union: mapping the regulatory basis, development, quality control, implementation and evaluation across member states. Health Policy 2012;107(2-3):146-56. https:// doi.org/10.1016/j.healthpol.2012.08.004

9. Cabana MD, Rand CS, Powe NR, Wu AW, Wilson MH, Abboud PA, et al. Why don't physicians follow clinical practice guidelines? A framework for improvement. JAMA 1999;282(15):1458-65.

10. Michie S, Johnston M, Francis J, Hardeman W, Eccles M. From Theory to Intervention: Mapping Theoretically Derived Behavioural Determinants to Behaviour Change Techniques. Appl Psychol 2008;57(4):660-80. https://doi.org/10.1111/j.1464-0597.2008.00341.x

11. McAuley L, Ramsey C. Cochrane effective practice and organisation of care review group; data collection checklist. Ottawa: EPOC, University of Ottawa; 2002. http://methods.cochrane.org/sites/methods.cochrane.org.bias/files/ public/uploads/EPOC\%20Data\%20Collection\%20Checklist.pdf Accessed 15.02.2018.

12. Grimshaw JM, Thomas RE, MacLennan G, Fraser C, Ramsay CR, Vale L, et al. Effectiveness and efficiency of guideline dissemination and implementation strategies. Health Technol Assess. 2004;8(6):iii-iv, 1-72.

13. Prior M, Guerin M, Grimmer-Somers K. The effectiveness of clinical guideline implementation strategies-a synthesis of systematic review findings. J Eval Clin Pract 2008;14(5):888-97. https://doi.org/10. $1111 /$ j.1365-2753.2008.01014.x

14. Grol R, Wensing M, Eccles MP, Davis D. Improving Patient Care: The Implementation of Change in Health Care Wiley-Blackwell Hoboken 2013.

15. Wensing $\mathbf{M}$, van der Weijden $\mathbf{T}$, Grol $\mathbf{R}$. Implementing guidelines and innovations in general practice: which interventions are effective? $\mathrm{Br} \mathrm{J}$ Gen Pract 1998;48(427):991-7.

16. Standard Country or Area Codes for Statistical Use. The United Nations Statistics Division https://unstats.un.org/unsd/methodology/m49/ overview/ Accessed 15.02.2018.

17. Liberati A, Altman DG, Tetzlaff J, Mulrow C, Gotzsche PC, Ioannidis JP, et al. The PRISMA statement for reporting systematic reviews and meta-analyses of studies that evaluate health care interventions: explanation and elaboration. PLoS Med 2009;6(7):e1000100. https://doi.org/ 10.1371/journal.pmed. 1000100

18. Ogilvie D, Fayter D, Petticrew M, Sowden A, Thomas S, Whitehead M, et al. The harvest plot: a method for synthesising evidence about the differential effects of interventions. BMC Med Res Methodol 2008;8:8. https://doi.org/10.1186/1471-2288-8-8

19. Higgins JPT, Green S, eds. Cochrane Handbook for Systematic Reviews of Interventions Version 5.1.0 [updated March 2011]. The Cochrane Collaboration, 2011. Available from http://handbook.cochrane.org Accessed 15.02.2018.

20. Eldridge S, Kerry S. A Practical Guide to Cluster Randomised Trials in Health Services Research. Chichester: Wiley; 2012.

21. Viechtbauer W. Conducting meta-analyses in $\{\mathrm{R}\}$ with the \{metafor package. J Stat Softw 2010;36(3):1-48.

22. $\mathrm{R}$ Core Team. A language and environment for statistical computing.: $\mathrm{R}$ Foundation for Statistical Computing, Vienna; 2014.

23. Egger M, Davey Smith G, Schneider M, Minder C. Bias in meta-analysis detected by a simple, graphical test. BMJ 1997;315(7109):629-34.

24. Aakhus E, Granlund I, Odgaard-Jensen J, Oxman AD, Flottorp SA. A tailored intervention to implement guideline recommendations for elderly patients with depression in primary care: a pragmatic cluster randomised trial. Implement Sci 2016;11:32. https://doi.org/10.1186/s13012-016-0397-3

25. Anderson D, Zlateva I, Khatri $\mathbf{K}$, Ciaburri N. Using health information technology to improve adherence to opioid prescribing guidelines in primary care. Clin J Pain 2015;31(6):573-9. https://doi.org/10.1097/ ajp.0000000000000177

26. Arts DL, Abu-Hanna A, Medlock SK, van Weert HC. Effectiveness and usage of a decision support system to improve stroke prevention in 
general practice: A cluster randomized controlled trial. PLoS One 2017;12(2):e0170974. https://doi.org/10.1371/journal.pone.0170974

27. Bermejo I, Schneider F, Kriston L, Gaebel W, Hegerl U, Berger M, et al. Improving outpatient care of depression by implementing practice guidelines: a controlled clinical trial. Int $J$ Qual Health Care 2009;21(1):29-36. https://doi.org/10.1093/intqhc/mzn050

28. Bonds DE, Hogan PE, Bertoni AG, Chen H, Clinch CR, Hiott AE, et al. A multifaceted intervention to improve blood pressure control: The Guideline Adherence for Heart Health (GLAD) study. Am Heart J 2009; 157(2):278-84. https://doi.org/10.1016/j.ahj.2008.09.021

29. Braun V, Heintze C, Rufer V, Welke J, Stein T, Mehrhof F, et al. Innovative strategy for implementing chronic heart failure guidelines among family physicians in different healthcare settings in Berlin. Eur $\mathrm{J}$ Heart Fail 2011;13(1):93-9. https://doi.org/10.1093/eurjhf/hfq181

30. Chen JH, Hom J, Richman I, Asch SM, Podchiyska T, Johansen NA Effect of opioid prescribing guidelines in primary care. Medicine 2016;95(35):e4760. https://doi.org/10.1097/md.0000000000004760

31. Dormuth CR, Carney G, Taylor S, Bassett K, Maclure M. A randomized trial assessing the impact of a personal printed feedback portrait on statin prescribing in primary care. J Contin Educ Heal Prof 2012;32(3):153-62. https://doi.org/10.1002/chp.21140

32. Ennis J, Gillen D, Rubenstein A, Worcester E, Brecher ME, Asplin J, et al. Clinical decision support improves physician guideline adherence for laboratory monitoring of chronic kidney disease: a matched cohort study. BMC Nephrol 2015;16:163. https://doi.org/10.1186/s12882015-0159-5

33. Franx G, Huyser J, Koetsenruijter $J$, van der Feltz-Cornelis CM, Verhaak PF, Grol RP, et al. Implementing guidelines for depression on antidepressant prescribing in general practice: a quasi-experimental evaluation. BMC Fam Pract 2014;15:35. https://doi.org/10.1186/ 1471-2296-15-35

34. French SD, McKenzie JE, O'Connor DA, Grimshaw JM, Mortimer D, Francis JJ, et al. Evaluation of a theory-informed implementation intervention for the management of acute low back pain in general medical practice: the IMPLEMENT cluster randomised trial. PLoS One 2013;8(6):e65471. https://doi.org/10.1371/journal.pone.0065471

35. Grunfeld E, Manca D, Moineddin R, Thorpe KE, Hoch JS, CampbellScherer $\mathbf{D}$, et al. Improving chronic disease prevention and screening in primary care: results of the BETTER pragmatic cluster randomized controlled trial. BMC Fam Pract 2013;14:175. https://doi.org/10.1186/ 1471-2296-14-175

36. Harris MF, Parker SM, Litt J, van Driel M, Russell G, Mazza D, et al. Implementing guidelines to routinely prevent chronic vascular disease in primary care: the Preventive Evidence into Practice cluster randomised controlled trial. BMJ Open 2015;5(12):e009397. https://doi.org/10. 1136/bmjopen-2015-009397

37. Heppe DB, Bucher-Bartelson B, Estacio RO, Krantz MJ. Impact of cardiovascular disease guideline dissemination on provider knowledge. Am J Med Sci 2012;343(1):56-60. https://doi.org/10.1097/MAJ. Ob013e31822817e6

38. Hunt JS, Siemienczuk J, Gillanders W, LeBlanc BH, Rozenfeld Y, Bonin $\mathbf{K}$, et al. The impact of a physician-directed health information technology system on diabetes outcomes in primary care: a pre- and postimplementation study. Inform Prim Care 2009;17(3):165-74.

39. Ioannidis G, Papaioannou A, Thabane L, Gafni A, Hodsman A, Kvern B, et al. The utilization of appropriate osteoporosis medications improves following a multifaceted educational intervention: the Canadian quality circle project (CQC). BMC Med Educ 2009;9:54. https://doi.org/10. 1186/1472-6920-9-54

40. Kiessling A, Lewitt M, Henriksson P. Case-based training of evidencebased clinical practice in primary care and decreased mortality in patients with coronary heart disease. Ann Fam Med 2011;9(3):211-8. https://doi.org/10.1370/afm. 1248

41. Licskai C, Sands T, Ong M, Paolatto L, Nicoletti I. Using a knowledge translation framework to implement asthma clinical practice guidelines in primary care. Int J Qual Health Care 2012;24(5):538-46. https://doi. org/10.1093/intqhc/mzs043

42. Liddy C, Hogg W, Singh J, Taljaard M, Russell G, Deri Armstrong C, et al. A real-world stepped wedge cluster randomized trial of practice facilitation to improve cardiovascular care. Implement Sci 2015;10:150. https://doi.org/10.1186/s13012-015-0341-y

43. Mahlknecht A, Abuzahra ME, Piccoliori G, Enthaler N, Eng1 A, Sonnichsen A. Improving quality of care in general practices by selfaudit, benchmarking and quality circles. Wien Klin Wochensch 2016;128(19-20):706-18. https://doi.org/10.1007/s00508-016-1064-Z
44. Mallen CD, Nicholl BI, Lewis M, Bartlam B, Green D, Jowett S, et al. The effects of implementing a point-of-care electronic template to prompt routine anxiety and depression screening in patients consulting for osteoarthritis (the Primary Care Osteoarthritis Trial): A cluster randomised trial in primary care. PLoS Med 2017;14(4):e1002273. https://doi.org/10.1371/journal.pmed. 1002273

45. Mold JW, Aspy CB, Smith PD, Zink T, Knox L, Lipman PD, et al. Leveraging practice-based research networks to accelerate implementation and diffusion of chronic kidney disease guidelines in primary care practices: a prospective cohort study. Implement Sci 2014;9:169. https://doi.org/10.1186/s13012-014-0169-x

46. Peters-Klimm F, Campbell S, Muller-Tasch T, Schellberg D, Gelbrich G, Herzog W, et al. Primary care-based multifaceted, interdisciplinary medical educational intervention for patients with systolic heart failure: lessons learned from a cluster randomised controlled trial. Trials 2009;10:68. https://doi.org/10.1186/1745-6215-10-68

47. Redaelli M, Vollmar HC, Simic D, Maly-Schurer C, Loscher S, Koneczny N. [Guideline implementation study on asthma: Results of a pragmatic implementation approach]. Z Evid Fortbild Qual Gesundhwes 2015;109(2):124-31. https://doi.org/10.1016/j.zefq.2015.04.015

48. Romera I, Montejo AL, Aragones E, Arbesu JA, Iglesias-Garcia C, Lopez S, et al. Systematic depression screening in high-risk patients attending primary care: a pragmatic cluster-randomized trial. BMC Psychiatry 2013;13:83. https://doi.org/10.1186/1471-244x-13-83

49. Secher N, Mikkelsen MM, Adelborg K, Mikkelsen R, Grove EL, Rubak JM, et al. Direct mail improves knowledge of basic life support guidelines in general practice: a randomised study. Scand J Trauma Resusc Emerg Med 2012;20:72. https://doi.org/10.1186/1757-7241-20-72

50. Shah BR, Bhattacharyya O, Yu CH, Mamdani MM, Parsons JA, Straus SE, et al. Effect of an educational toolkit on quality of care: a pragmatic cluster randomized trial. PLoS Med 2014;11(2):e1001588. https://doi. org/10.1371/journal.pmed. 1001588

51. Sinnema H, Majo MC, Volker D, Hoogendoorn A, Terluin B, Wensing $\mathbf{M}$, et al. Effectiveness of a tailored implementation programme to improve recognition, diagnosis and treatment of anxiety and depression in general practice: a cluster randomised controlled trial. Implement Sci 2015;10:33. https://doi.org/10.1186/s13012-015-0210-8

52. Sipila R, Helin-Salmivaara A, Korhonen MJ, Ketola E. Change in antihypertensive drug prescribing after guideline implementation: a controlled before and after study. BMC Fam Pract 2011;12:87. https:// doi.org/10.1186/1471-2296-12-87

53. Skoglund I, Bjorkelund C, Petzold M, Gunnarsson R, Moller M. A randomized controlled trial comparing two ways of providing evidencebased drug information to GPs. Scand J Prim Health Care 2013;31(2):6772. https://doi.org/10.3109/02813432.2012.757071

54. Soler N, Ballester E, Martin A, Gobartt E, Miravitlles M, Torres A. Changes in management of chronic obstructive pulmonary disease (COPD) in primary care: EMMEPOC study. Respir Med 2010;104(1):6775. https://doi.org/10.1016/j.rmed.2009.08.003

55. Tahvonen $\mathbf{P}$, Oikarinen $\mathbf{H}$, Niinimaki J, Liukkonen E, Mattila $\mathbf{S}$, Tervonen O. Justification and active guideline implementation for spine radiography referrals in primary care. Acta Radiol 2017;58(5):586-92. https://doi.org/10.1177/0284185116661879

56. Valles-Fernandez R, Rodriguez-Blanco T, Mengual-Martinez L, RosellMurphy M, Prieto-De Lamo G, Martinez-Frutos F, et al. Intervention for control of hypertension in Catalonia, Spain (INCOTECA Project): results of a multicentric, non-randomised, quasi-experimental controlled intervention study. BMJ Open 2012;2(2):e000507. https://doi.org/10. 1136/bmjopen-2011-000507

57. Verbiest ME, Presseau J, Chavannes NH, Scharloo M, Kaptein AA, Assendelft WJ, et al. Use of action planning to increase provision of smoking cessation care by general practitioners: role of plan specificity and enactment. Implement Sci 2014;9:180. https://doi.org/10.1186/ s13012-014-0180-2

58. Vidal-Pardo JI, Perez-Castro TR, Lopez-Alvarez XL, Santiago-Perez MI, Garcia-Soidan FJ, Muniz J. Effect of an educational intervention in primary care physicians on the compliance of indicators of good clinical practice in the treatment of type 2 diabetes mellitus [OBTEDIGA project]. Int J Clin Pract 2013;67(8):750-8. https://doi.org/10.1111/ijcp. 12145

59. Wentworth AL, Fox CH, Kahn LS, Glaser K, Cadzow R. Two years after a quality improvement intervention for chronic kidney disease care in a primary care office. Am J Med Qual 2011;26(3):200-5. https://doi.org/ $10.1177 / 1062860610381916$

60. Giguere A, Legare F, Grimshaw J, Turcotte S, Fiander M, Grudniewicz A, et al. Printed educational materials: effects on professional practice and 
healthcare outcomes. Cochrane Database Syst Rev 2012;10:CD004398. https://doi.org/10.1002/14651858.CD004398.pub3

61. Forsetlund L, Bjorndal A, Rashidian A, Jamtvedt G, O'Brien MA, Wolf F, et al. Continuing education meetings and workshops: effects on professional practice and health care outcomes. Cochrane Database Syst Rev. 2009(2):Cd003030. https://doi.org/10.1002/14651858.CD003030.pub2

62. Francke AL, Smit MC, de Veer AJ, Mistiaen P. Factors influencing the implementation of clinical guidelines for health care professionals: a systematic meta-review. BMC Med Inform Decis Mak 2008;8:38. https:// doi.org/10.1186/1472-6947-8-38

63. Unverzagt S, Oemler M, Braun K, Klement A. Strategies for guideline implementation in primary care focusing on patients with cardiovascular disease: a systematic review. Fam Pract 2014;31(3):247-66. https://doi. org/10.1093/fampra/cmt080

64. Ivers N, Jamtvedt G, Flottorp S, Young JM, Odgaard-Jensen J, French SD, et al. Audit and feedback: effects on professional practice and healthcare outcomes. Cochrane Database Syst Rev. 2012(6):Cd000259. https://doi.org/10.1002/14651858.CD000259.pub3

65. Shojania KG, Jennings A, Mayhew A, Ramsay CR, Eccles MP, Grimshaw J. The effects of on-screen, point of care computer reminders on processes and outcomes of care. Cochrane Database Syst Rev. 2009(3):Cd001096. https://doi.org/10.1002/14651858.CD001096. pub2

66. Arditi C, Rege-Walther M, Wyatt JC, Durieux P, Burnand B. Computergenerated reminders delivered on paper to healthcare professionals; effects on professional practice and health care outcomes. Cochrane Database Syst Rev. 2012;12:Cd001175. https://doi.org/10.1002/ 14651858.CD001175.pub3

67. Squires JE, Sullivan K, Eccles MP, Worswick J, Grimshaw JM. Are multifaceted interventions more effective than single-component interventions in changing health-care professionals' behaviours? An overview of systematic reviews. Implement Sci 2014;9:152. https://doi.org/10. 1186/s13012-014-0152-6
68. Solberg LI. Guideline implementation: what the literature doesn't tell us. Jt Comm J Qual Improv 2000;26(9):525-37.

69. Nilsen P. Making sense of implementation theories, models and frameworks. Implement Sci 2015;10:53. https://doi.org/10.1186/s13012015-0242-0

70. Davies P, Walker AE, Grimshaw JM. A systematic review of the use of theory in the design of guideline dissemination and implementation strategies and interpretation of the results of rigorous evaluations. Implement Sci 2010;5: 14. https://doi.org/10.1186/1748-5908-5-14

71. Baker R, Camosso-Stefinovic J, Gillies C, Shaw EJ, Cheater F, Flottorp S, et al. Tailored interventions to address determinants of practice. Cochrane Database Syst Rev. 2015(4):Cd005470. https://doi. org/10.1002/14651858.CD005470.pub3

72. Hoomans T, Evers SM, Ament AJ, Hubben MW, van der Weijden T, Grimshaw JM, et al. The methodological quality of economic evaluations of guideline implementation into clinical practice: a systematic review of empiric studies. Value Health 2007;10(4):305-16. https://doi.org/10. $1111 /$ j.1524-4733.2007.00175.x

73. Brouwers MC, Kho ME, Browman GP, Burgers JS, Cluzeau F, Feder G, et al. AGREE II: advancing guideline development, reporting, and evaluation in health care. Prev Med 2010;51(5):421-4. https://doi.org/ 10.1016/j.ypmed.2010.08.005

74. Flodgren G, Hall AM, Goulding L, Eccles MP, Grimshaw JM, Leng GC, et al. Tools developed and disseminated by guideline producers to promote the uptake of their guidelines. Cochrane Database Syst Rev. 2016(8):Cd010669. https://doi.org/10.1002/14651858.CD010669.pub2

75. Krahn M, Naglie G. The next step in guideline development: incorporating patient preferences. JAMA 2008;300(4):436-8. https://doi.org/10. 1001/jama.300.4.436

76. Hajjaj FM, Salek MS, Basra MK, Finlay AY. Non-clinical influences on clinical decision-making: a major challenge to evidence-based practice. J R Soc Med 2010;103(5):178-87. https://doi.org/10.1258/jrsm.2010. 100104 